\title{
Experimental investigation of the low-frequency impact sound transfer function of lightweight wooden floors via heel drops
}

\author{
Yi Qin, Jin Jack Tan, and Maarten Hornikx* \\ Eindhoven University of Technology, 5612 Eindhoven, The Netherlands
}

Received 24 February 2020, Accepted 29 September 2020

\begin{abstract}
The lightweight floor is a popular construction choice in buildings despite its poor low-frequency impact sound performance. This is exacerbated by common human activities, such as walking and jumping, that have high input force levels at low frequencies. Therefore, experimental evaluations of the low-frequency impact sound performance of a wooden lightweight floor are of interest to designers and researchers. The aim of this paper is to explore the use of heel drop for impact sound transfer measurement. An impact force plate has been built to accurately measure the heel-drop forces up to $200 \mathrm{~Hz}$ and the performances on two types of floors are evaluated. The heel drop has a higher energy level at low frequencies, resulting in higher coherence and signal-to-noise ratio and hence superior performance in characterizing the floors in the frequency range of below $40 \mathrm{~Hz}$ when compared to the impact hammer. Interestingly, in the case of a heel-drop excitation, the first natural frequencies of the floor decrease. It is in contrast to when a human is simply standing on it, which resulted in an increase of the natural frequencies upon impact hammer excitation.
\end{abstract}

Keywords: Low-frequency impact sound, Lightweight floor, Heel drop, Transfer function, Floating floor

\section{Introduction}

Lightweight wooden floors (LWF) have poor sound insulation in the low-frequency range $(<200 \mathrm{~Hz})$ due to their light weight and low rigidity [1, 2]. Unfortunately, most of the real-life impacts induced by human activities, like walking and jumping, have a large amount of energy at such frequencies. This could lead to noise annoyance for occupants living in wooden-based houses [2-4] and the low-frequency impact sound of LWFs remains a major concern for consumers and researchers [5-7]. In the lowfrequency range, both the floor and the room have several natural modes dominating their responses $[8,9]$. One popular method to understand the low-frequency behavior of LWFs is experimental modal analysis [5, 10], i.e. the floor is excited by an impact hammer or a shaker with known amplitude and is characterized via transfer functions. The results of the transfer function provide the magnitude and the phase of the floor's responses over a frequency range and show the natural frequencies that are more sensitive to excitations [5]. Based on that, further studies on reducing the impact disturbance or optimizing the floor design can be carried out, such as by comparing prediction models and modal analysis methods [7, 9].

However, experimental modal analyses were not amply extended to the impact sound field. Most of the studies on

*Corresponding author: m.c.j.hornikx@tue.nl impact sound are based on the measurement of sound pressure levels excited by the standard impact sources, e.g., the ISO tapping machine and rubber ball $[1,6]$. This provides a way to compare the performances of various floors, but it does not intuitively reflect the ability of a structure to radiate sound as the force level is included in this value $[1,6]$. In comparison, the transfer function allows a evaluation on the behavior of a floor-room system, and provides a convenient way to investigate the sensitivity of the floor-room system to generate noise at low frequencies $[9,11]$.

The conventional impact sources for floor modal testing, e.g., impact hammer and shaker, have been applied in the measurement of structure-borne sound in buildings [11]. The latter assures high-quality modal tests, while the former allows convenient in-situ floor structure measurements [12]. However, the sound generated by these sources tends to have a low signal-to-noise ratio in the low-frequency range [11]. Moreover, they cannot provide an acoustical scenario close to those given by the human activities $[2,13]$, as the floor responds differently due to its interaction with humans.

On the other hand, human-induced impacts, such as the heel drop, gives a higher excitation level at low frequencies and resembles the floor-occupant situation in real life, as has been explored in researches on the low-frequency response of floor structures $[5,13,14]$. With the assistance of a force plate measurement device, the forces of heel-drop 
impacts can be measured during response testing, which makes the calculation of transfer functions possible. Currently, this method has been explored in a measurement of the floor mobility as an alternative method to evaluate the floor vibrational performance for engineering purposes [5]. However, the work failed to include the effects of heel drop in terms of transfer function.

In this paper, the low-frequency modal behavior of a LWF-room system is studied. As a commonly-used measure to improve the impact sound insulation, a concrete floating floor (CFF) was built on the LWF and the response function of the coupled structure was also investigated. The two objectives of this paper are as follows: (1) to experimentally investigate impact sound performance of LWF and LWF + CFF by means of transfer function instead of sound pressure and (2) to investigate the heel-drop test as an improved method, compared to traditional impact-hammer excitation methods, for the impact-sound transfer-function measurement on lightweight floors. To achieve these two objectives, two types of impact sources, the impact hammer and the heel drop, were used. Additionally, the effect of the human body weight on the natural mode behavior of the floor and the room were also analyzed. The paper is organized as follows. Section 2 describes the experiment and the impact force plate device used for the heel-drop measurement. Section 3 presents the results of transfer function measurements on the floor. The results are discussed in Section 4 and conclusions can be found in Section 5 .

\section{Methodology of the measurement}

\subsection{Floor construction}

A $3.35 \times 3.35 \mathrm{~m}^{2}$ wooden floor was constructed and installed as the ceiling of the acoustic transmission suite in the Eindhoven University of Technology as shown in Figure 1.

The lightweight wooden floor was built according to the reference floor C2 as specified in ISO 10140-5 [15]. The top surface of the floor is made up of six pieces of plywood plates, and they are mainly supported by seven spruce joists with a height of $190 \mathrm{~mm}$ and a width of $45 \mathrm{~mm}$ along one direction. The joists were spaced with an equal distance of $413 \mathrm{~mm}$ (see Fig. 2a). The top plates are joined by "tongue and groove" connections and are secured to the joists with nails ( 25 nails $/ \mathrm{m}$ ). The whole floor is supported on four edges by the joists with the same dimension as the seven joists in the middle. All edge joists are resting on a concrete floor with a thickness of $340 \mathrm{~mm}$.

The second investigated floor is a concrete floating floor that is built on top of the aforementioned wooden floor. It is first made up of a $30 \mathrm{~mm}$ thick continuous rock wool resilient layer and on top of it a $60 \mathrm{~mm}$-thick concrete layer, as shown in Figure 2b. These two layers cover the surface area of the wooden floor completely. The floating floor is terminated with elastic foam at its edges to minimize vibration transmission from the concrete plate to the wooden floor. The material properties of these two floors are summarized in Table 1. The material properties of the wood and

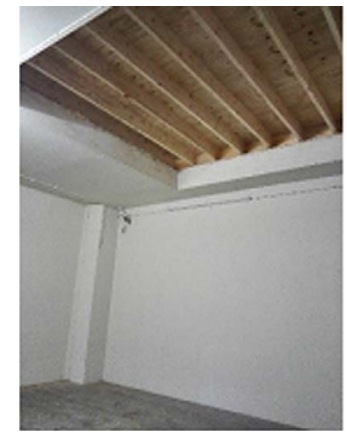

(a)

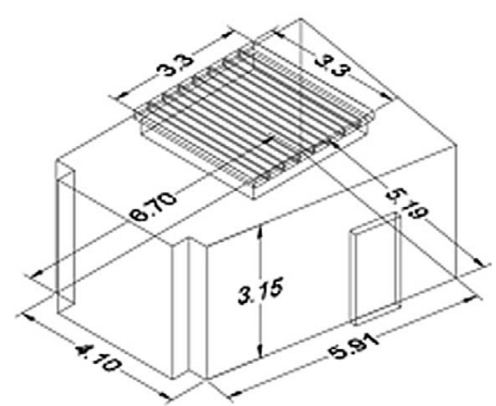

(b)
Figure 1. Transmission room $\left(98 \mathrm{~m}^{3}\right)$ at Eindhoven University of Technology. (a) Experimental setup in the transmission room. (b) Dimensions of the transmission room.

concrete were characterized via modal testing on the materials with an impact hammer. The properties of the rock wool were obtained from the product specification.

\subsection{Measurement setup}

The measurement setup can be divided into two parts. First, the vibration of the floors is measured by 12 accelerometers (PCB 333B30); and secondly, the sound pressure in the receiving chamber is measured by four microphones (PCB 378B02) with their positions shown in Figure 3 . The microphones 1 and 2 are placed close to the corners of the room at the height of 1.25 and $1.5 \mathrm{~m}$; microphone 3 and microphone 4 are placed under the floor at height of 1.75 and $2 \mathrm{~m}$. Two impact positions (one joist excitation and one inter-joist excitation) are chosen at the place that can excite the modes at low frequencies. To complement that, accelerometers are distributed along the $x$ and $y$-direction of the impact position to not only measure the responses but at the same time to identify the mode shapes of the floor along two directions. To observe the behaviors of different floor modes, four accelerometer positions are chosen alongside the impact position to present the floor response and their positions are set more than $0.7 \mathrm{~m}$ apart from the impact position to minimize the effect of the impact.

Two types of impact sources are used: an impact hammer (BK 8202) with a rubber tip and the heel drop given by a human. The experimenter who performs the heel-drop excitation has a bodyweight of $80 \mathrm{~kg}$, while the measurement of the body load effect (Sect. 3.1.4) was performed with a standing experimenter of $72 \mathrm{~kg}$. Careful considerations were given such that impact point or the gravity center of the human lies either on the joist or in the midpoint between two joists. To avoid that the accelerometer and the impact are at the same locations (driving-point measurement with hammer impacts in Sect. 3.1), the receiver is placed around $1 \mathrm{~cm}$ away from the impact positions instead.

The signals were captured by a National-Instruments acquisition system (NI 9234 and cDAQ-9178) for $7 \mathrm{~s}$ with 


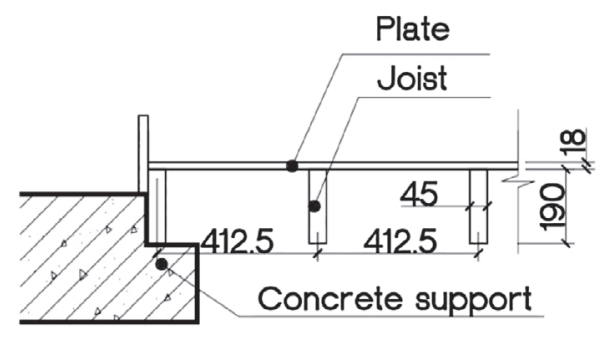

(a) LWF

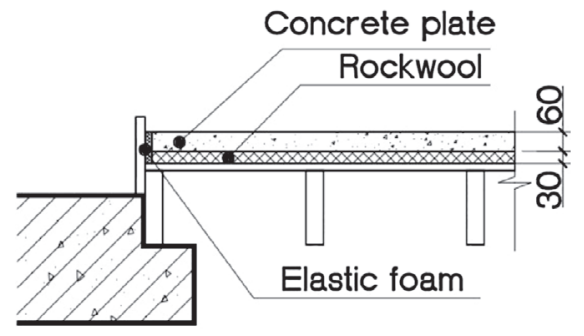

(b) $\mathrm{LWF}+\mathrm{CFF}$

Figure 2. Cross sections of the floor investigated. Dimensions in mm. (a) LWF, (b) LWF + CFF.

Table 1. Material properties of the floors.

\begin{tabular}{lcccc}
\hline & \multicolumn{2}{c}{ Wooden base floor } & \multicolumn{2}{c}{ Floating floor } \\
\cline { 2 - 4 } & Plate & Joist & & Floating plate \\
\hline Material & Plywood & Spruce & Concrete & Rosilient layer \\
Average density $\left(\mathrm{kg} / \mathrm{m}^{3}\right)$ & 520 & 430 & 2080 & 100 \\
Modulus of elasticity $(\mathrm{GPa})$ & 4.6 & 9.3 & 27.9 & - \\
Stiffness $\left(\mathrm{MN} / \mathrm{m}^{3}\right)$ & - & - & - & 11 \\
\hline
\end{tabular}

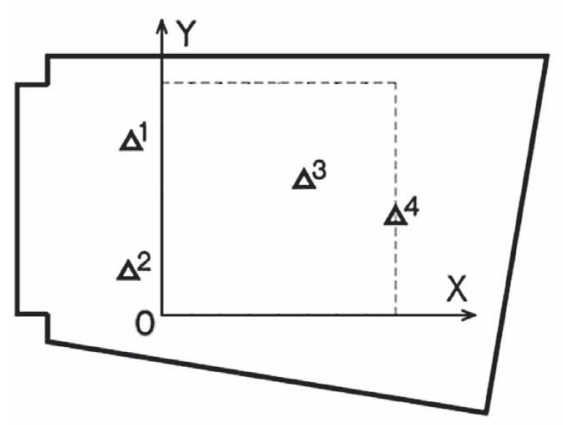

(a)

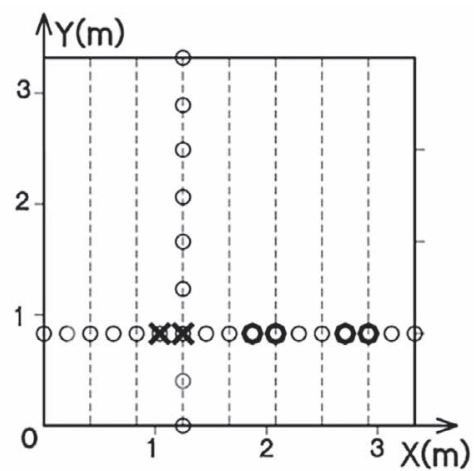

Impact positions

Accelerometers for mode shape characterization

- Accelerometers for transfer-mobility comparison

$\Delta$ microphones

Figure 3. (a) Top view of the room and (b) the floor, and positions of the source and receivers.

a sampling frequency of $51.2 \mathrm{kHz}$, and then the fast Fourier transform (FFT) was used to process the full signal with a rectangular window in Matlab up to $25.6 \mathrm{kHz}$.

\subsection{Impact force plate (IFP)}

To characterize and measure the forces exerted in a heel-drop excitation, an impact force plate was fabricated with the design shown in Figure 4 . The design and fabrication of the IFP are mainly due to the lack of existing devices capable of measuring the forces induced by a heel drop. The device has three force sensors (PCB 201B03) sandwiched by two triangular plates $(2 \mathrm{~mm}$ aluminum $+26 \mathrm{~mm}$ PVC foam $+2 \mathrm{~mm}$ aluminum) as shown in Figure 5 .
It is constructed to provide rigidity, low mass $(7.4 \mathrm{~kg})$ and the desired vibrational properties. Specific attention has been given in assuring that the IFP's own first mode is higher than $250 \mathrm{~Hz}$, making it suitable for measurements below $200 \mathrm{~Hz}$. The size of the plate is assumed to be much smaller than the bending wavelength in the wood material in the interested frequency range, such that the forces transmitted through the device can properly represent the point impact forces inserted by the human body into the floor. The device can be operated by simply placing it on top of the test floor. An illustration is given on the top plate to assist the placement of the experimenter to minimize the variance in the actual excitation position in repeating excitations. 


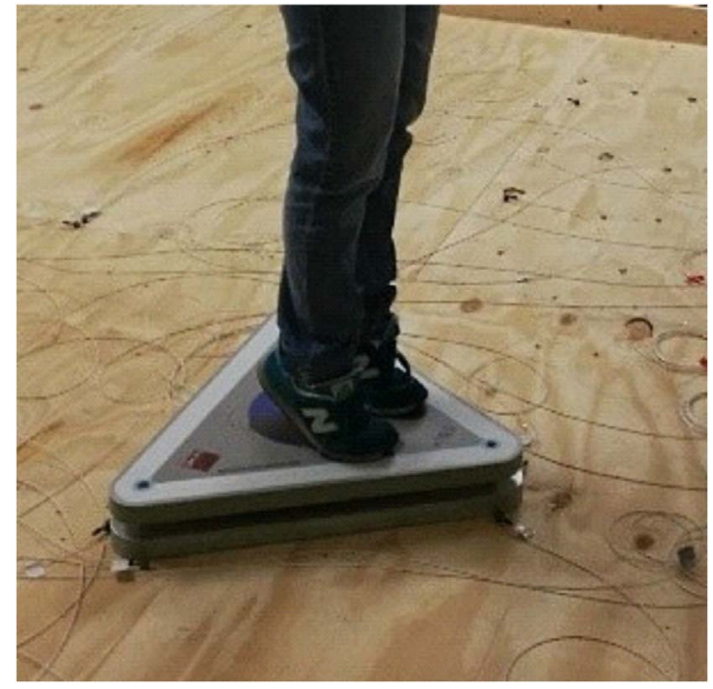

Figure 4. Impact force plate and heel-drop measurement.

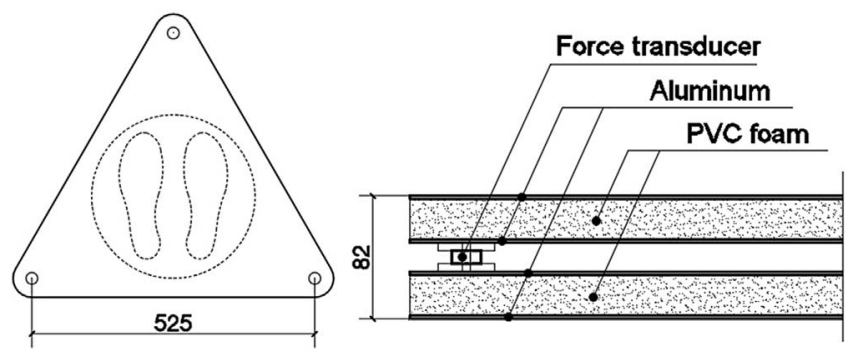

Figure 5. Schematic and cross section of the impact force plate. Dimensions in $\mathrm{mm}$.

The IFP is calibrated by exciting it 12 times with an impact hammer and comparing the input hammer force to the output force, i.e. the summed forces for all three transducers. The ratios of the root-mean-square values of the output forces (IFP) to that of the input forces (impact hammer) are calculated for the one-third-octave bands from $16 \mathrm{~Hz}$ to $200 \mathrm{~Hz}$, as shown in Figure 6. The IFP is placed on a $300 \mathrm{~mm}$ thick concrete floor during the calibration process to minimize the influence of floor vibrations on the measured input force. A ratio of 1.72 , determined by comparing the energies of the output and input forces below $160 \mathrm{~Hz}$, was used as the calibration factor of the IFP. At $200 \mathrm{~Hz}$, the IFP still responds linearly against hammer forces but with a higher output-input ratio, as affected by its first mode at $315 \mathrm{~Hz}$. However, to make the analysis simpler, the ratio of 1.72 is used and caution needs to be considered when analyzing results close to $200 \mathrm{~Hz}$. In that regard, the input force would be underestimated and hence the transfer function overestimated.

The calibration exercise is further extended by exciting the IFP with a free-falling basketball. The basketball was let free from the heights of $20,40,60,80$ and $100 \mathrm{~cm}$ with reference to the IFP. The average impact force, $F_{\text {pred }}$ was calculated via Equation (1) from the impulse-momentum theorem, where $m$ is the basketball mass, $h_{0}$ is the

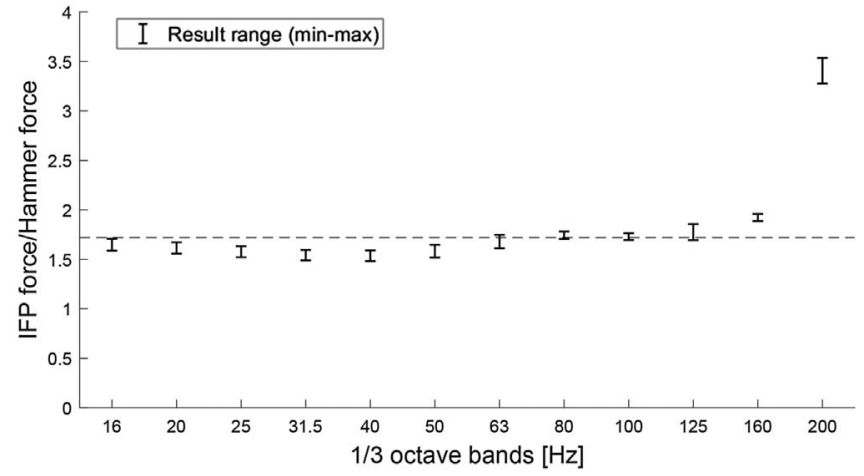

Figure 6. Ratios between the forces measured by IFP and impact-hammer for one-third-octave bands.

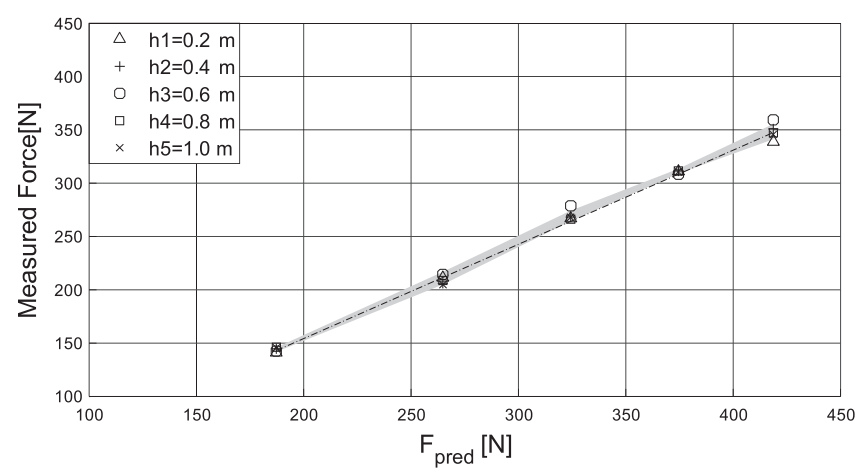

Figure 7. Comparison between calculated and measured basketball-dropping impact forces. Shaded area shows the confidence intervals of the measurements.

descending height, $r_{b}$ is the bounce height ratio and $\Delta t$ is the impact time (around $0.0105 \mathrm{~s}$ ) measured by the IFP; it is then compared with the RMS force measured by the IFP as shown in Figure 7. The IFP-measured force displays linear proportionality to the calculated force, showing that IFP responds linearly even when forces larger than the hammer are applied:

$$
F_{\text {pred }}=\frac{m\left(1+\sqrt{r_{b}}\right) \sqrt{2 g h_{0}}}{\Delta t} .
$$

\subsection{Processing of experimental results}

For each measurement setup, the impact force $f$, acceleration $a$, and sound pressure $p$ are first obtained as time signals before they are transformed to the frequency domain $F, A$ and $P$ via FFT. In the frequency domain, the velocity and displacement of the floor are retrieved from the relationships $V=A /(j \omega)$ and $U=A /(j \omega)^{2}$. The floor mobility $Y$ and the impact sound pressure transfer function $H_{P}$ are estimated by Equations (2a) and (2b), with $V, P$ and $F$ being averaged over six measurements and for all measurement points:

$$
\begin{gathered}
Y=V / F, \\
H_{P}=P / F .
\end{gathered}
$$


The results of the impact-sound transfer function are also described in term of one-third-octave band levels. First, the sound pressure level $L_{P r, s, n}$ is calculated for each measured output signals with a reference pressure of $2 \times 10^{-5} \mathrm{~Pa}$. The subscripts $r, s$ and $n$ represent the receiver position, impact position and the number of measurements, respectively. Similarly, the impact force level $L_{F s, n}$ is calculated for the input signals with a reference value of $10^{-6} \mathrm{~N}$, and the transfer function level of the impact sound $D_{P r, s, n}$ is defined by:

$$
D_{P r, s, n}=L_{P r, s, n}-L_{F s, n} .
$$

Then, the receiver-average transfer function level, $D_{P_{s, n}}$, from $R$ positions is given by:

$$
D_{P s, n}=10 \lg \left(\frac{\sum_{r=1}^{R} 10^{0.1 D_{P r, s, n}}}{R}\right) .
$$

Lastly, the transfer function levels are averaged over $S$ excitation positions and $N$ measurements:

$$
D_{P \text { avr }}=20 \lg \left(\frac{\sum_{s=1}^{S} \sum_{n=1}^{N} 10^{0.05 D_{P, n}}}{S \times N}\right) .
$$

\section{Results}

\subsection{Floor characterization}

The vibration of the two types of floors presented in Section 2.1 is first characterized by an impact hammer. The mode shapes of the lightweight wooden floor with and without the concrete floating floor (abbreviated as $\mathrm{LWF}+\mathrm{CFF}$ and LWF respectively) are inspected to provide a baseline to subsequent more complex measurements. During this measurement, the experimenter is not present on the test floor.

\subsubsection{Lightweight wooden floor (LWF)}

Figure 8 shows the displacement of the plate normalized by the force measured (a) perpendicular to the joists over 17 points and (b) along the joists over 9 points. The accelerometers are evenly spaced and placed on the top surface along the lines (a) $y=0.84 \mathrm{~m}$ and (b) $x=1.26 \mathrm{~m}$ respectively, and the impact position is at the intersection of the two lines as shown in Figure 3. In Figure 8a, the first few modes are clearly identified at $30,34,37,46$ and $52 \mathrm{~Hz}$ respectively with an increasing number of peaks along the $x$-direction. These modes are denoted as the $(1,1),(2,1),(3,1),(4,1)$ and $(5,1)$ modes respectively, where $(m, n)$ indicates that the mode has a mode number $m$ along the $x$-direction and $n$ along the $y$-direction. The numbering order of the modes follows the methods in [16]. The frequency of the modes is first identified by observing the amplitude and the phase shift of the signals [17]. The mode shapes and the boundary conditions were then determined visually. The first mode with two peaks in the $y$-direction is identified at around
$96 \mathrm{~Hz}$ as shown in Figure 8b. However, this would essentially be the $(4,2)$ mode, which has a mode shape with four peaks in the $x$-direction. The other modes, namely $(1,2)$, $(2,2)$ and $(3,2)$ are likely to be obscured by the other aforementioned modes. Nevertheless, comparing the frequencies of modes $(4,2)$ and $(2,1)$ shows that the mode number along the $y$-direction increases slower than that along the $x$-direction. One can tell that the joists provide a large stiffness to the lightweight floor along the $y$-direction. Overall, all identified mode shapes indicate that the setup exhibits simply-supported boundary conditions, as the displacement approaches zero at the boundaries.

\subsubsection{Lightweight wooden floor with concrete floating floor ( $L W F+C F F)$}

For the LWF $+\mathrm{CFF}$, the accelerometers are directly attached on top of the concrete floating floor as well as to the bottom surface. Due to the configuration at the boundaries of the bottom surface, the two points at both ends along the $x$-direction cannot be measured from the bottom. For the accelerometer positions that would coincide with the joists, these accelerometers are attached directly to the bottom flooring as close to the joist as possible, instead of at the bottom of the joist. The amplitude of the displacement normalized by the force is shown in Figure 9 .

Due to the mass added by the concrete plate onto the LWF, the new coupled floor system has a first mode around $19 \mathrm{~Hz}$ instead of $30 \mathrm{~Hz}$. The five identified modes and their corresponding mode shapes are summarized in Table 2. One can see that the measurements at the concrete floating layer exhibit mostly free boundary conditions due to the presence of the elastic layer. Additionally, the LWF follows the vibrational patterns of the upper layer due to the heavy mass of the latter, especially in the direction across the joists ( $x$-direction in Fig. 9a). In this direction, the lightweight composite structure has a lower stiffness compared to the other direction and as a result the vibrations of both layers have similar amplitudes. In any case, the experimental results illustrate the complexity of the coupled LWF + CFF system which can only be resolved in future investigation.

\subsubsection{Joist and inter-joist excitation}

On lightweight floor structures, a significant difference can be found in the driving-point mobility between the measurements at joist and inter-joist positions $[11,18]$. This is also observed in the current work, as shown in Figure 10a, affecting only the LWF but hardly the LWF + CFF (see the impact positions in Fig. 3). On the other hand, this discrepancy between the joist and inter-joist excitation is less pronounced in the transfer mobility and impact sound transfer function as shown in Figures 10 and 11 (a similar result was observed by [11]). For these two parameters, obvious deviations are only observed from $160 \mathrm{~Hz}$ onward. In the following measurements, the results for the comparison are averaged over both the joist and inter-joist 


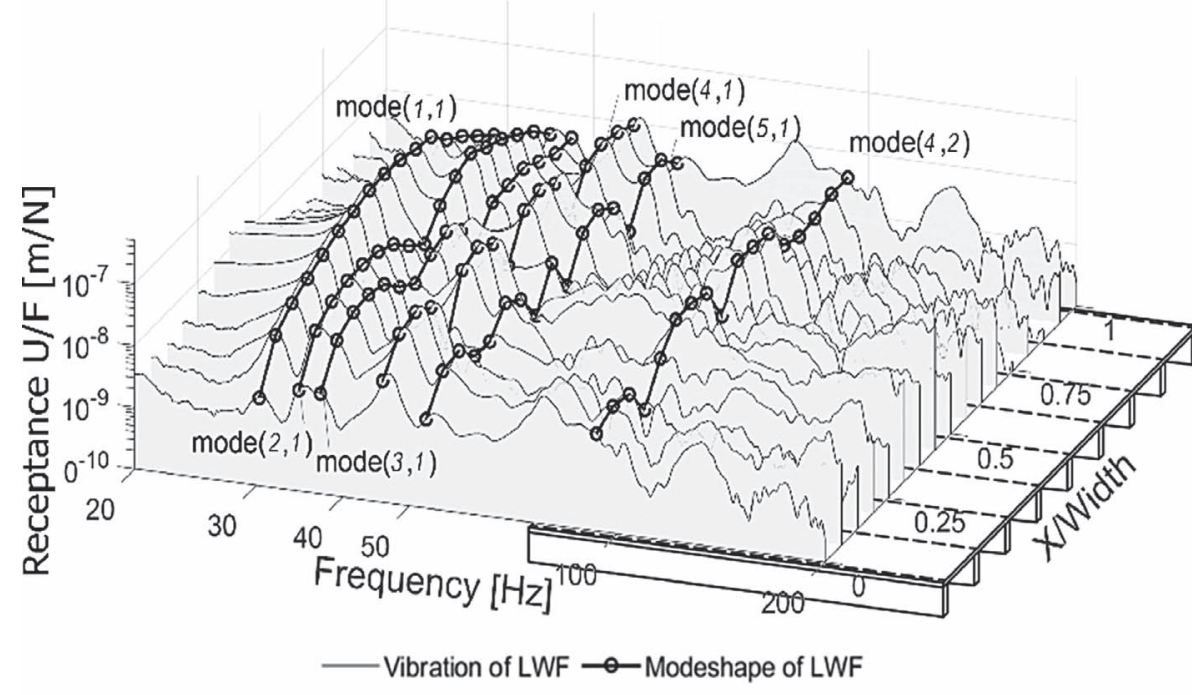

(a)

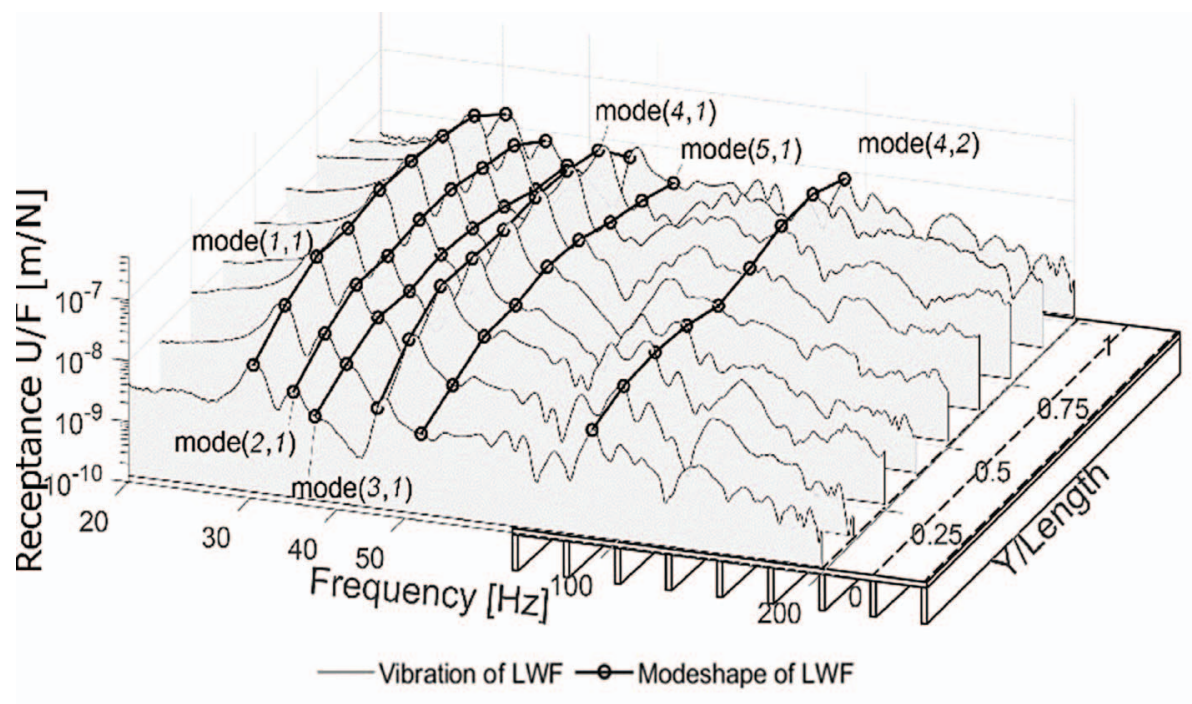

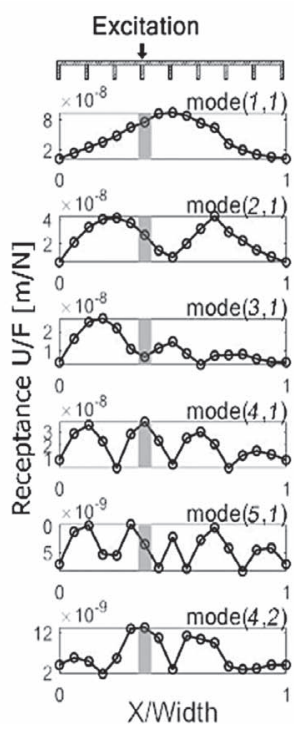

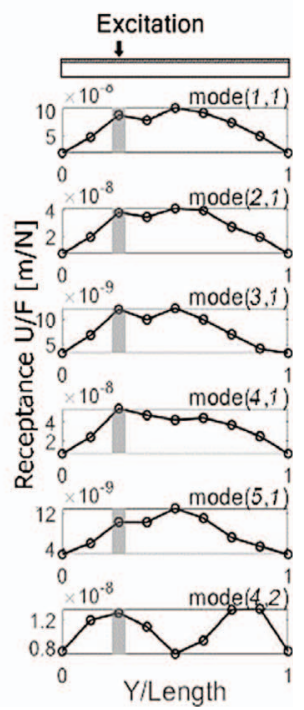

(b)

Figure 8. Vibrations and mode shapes of LWF. (a) Direction perpendicular to the joist. (b) Direction along the joist.

excitations in order to give the consideration on both types of excitation.

\subsubsection{Effect of body load}

The LWF and LWF + CFF are excited by the modal hammer while a $72 \mathrm{~kg}$ experimenter is standing upright on them close to the impact position. Figure 12 shows the transfer mobility when both types of flooring are excited by the hammer with and without a human standing upright on them. Two observations can be made on the effect of the body load on the LWF. First, the body load acts as a source of attenuation, reducing the mobility level by around $10 \mathrm{~dB}$ across the whole frequency range of interest. Secondly, the first four modes of the LWF increase in frequency by an average of $2 \mathrm{~Hz}$. This result is consistent with other literature findings [20,21], indicating a potential increase in the damping and stiffness of a coupled floor-human system in the interested frequency range compared to the floor-only system. On the LWF $+\mathrm{CFF}$, due to the mass added by the concrete layer, neither attenuation nor mode shift can be observed despite the presence of a body load.

Similar results are also observed in the measured impact sound pressure as shown in Figure 13. An attenuation around $10 \mathrm{~dB}$ and upward shifts of the natural frequencies are both observed in the LWF measurement and expectedly, only the floor modes (and not the room modes) are 


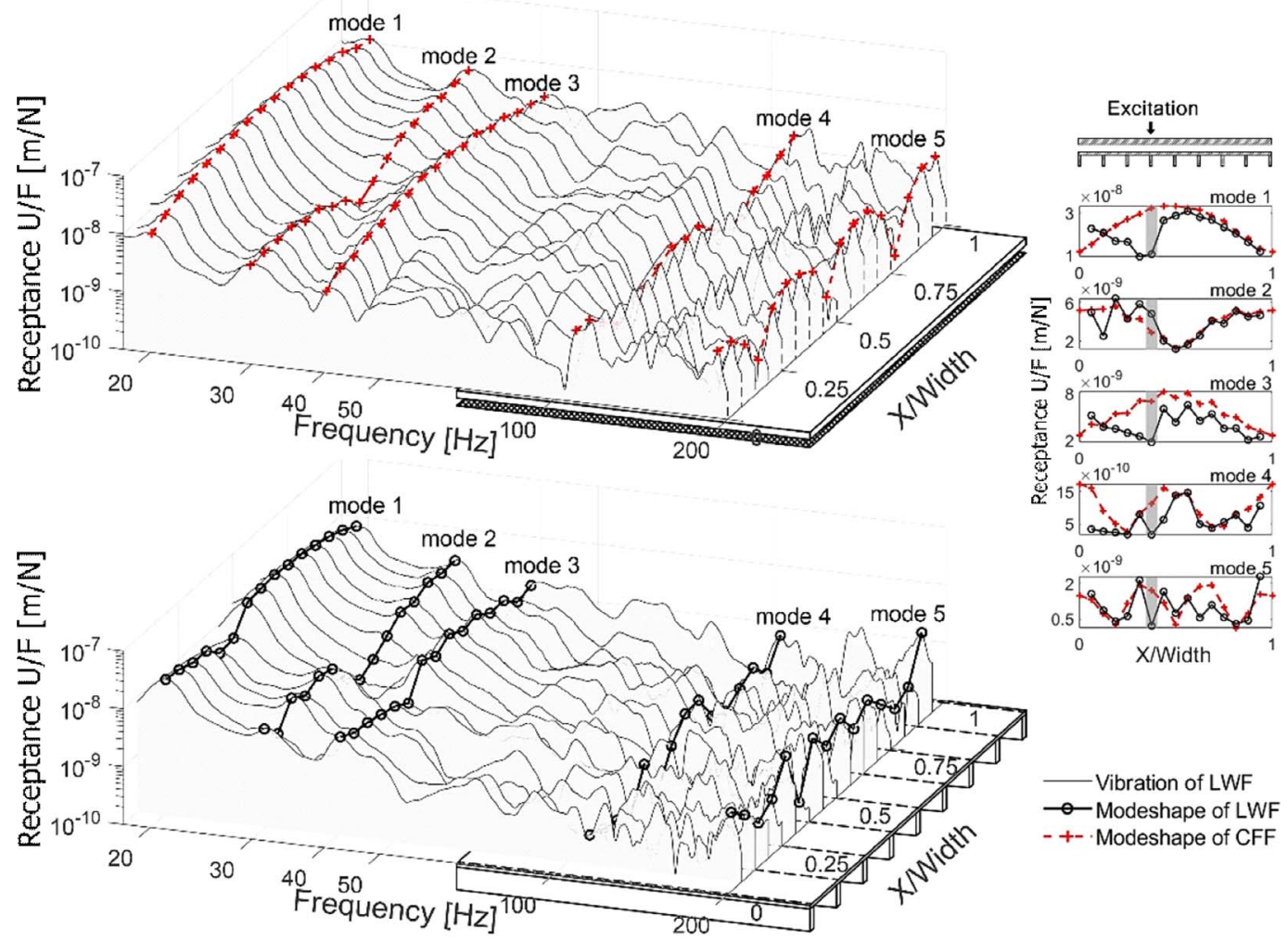

(a)

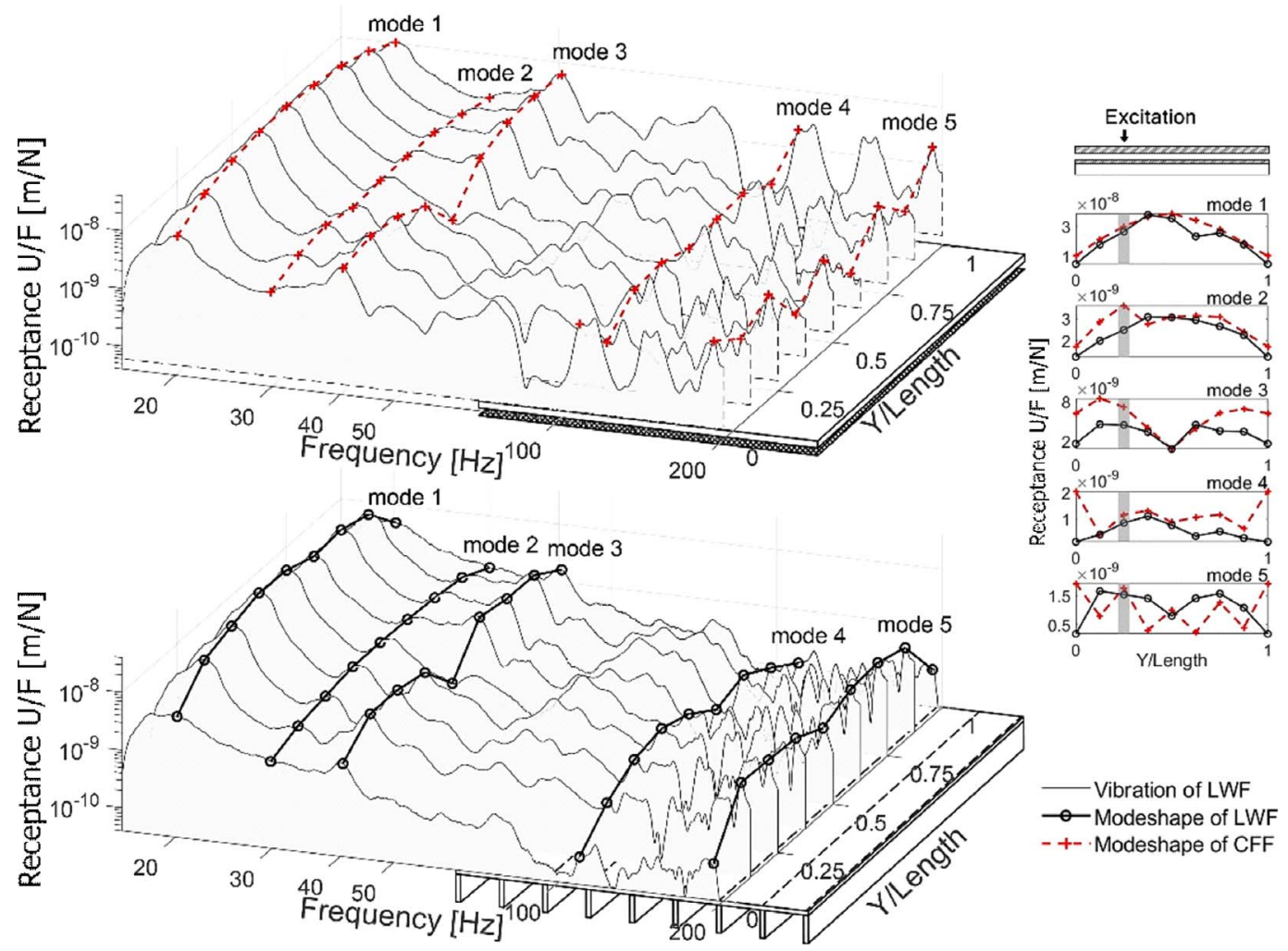

(b)

Figure 9. Vibrations and mode shapes of LWF + CFF. (a) Direction perpendicular to the joist. (b) Direction along the joist. 
Table 2. Mode shapes of LWF + CFF.

\begin{tabular}{lccc}
\hline Mode & Frequency $(\mathrm{Hz})$ & Wooden base floor of LWF + CFF & Concrete floating layer of LWF + CFF \\
\hline 1 & 19 & $\left(1 s^{*}, 1 s\right)$ & $(1 s, 1 s)$ \\
2 & 28 & $(0 f, 1 s)$ & $(0 f, 1 s)$ \\
3 & 40 & $\left(1 s^{*}, 2 s\right)$ & $(1 s, 0 f)$ \\
4 & 110 & $(1 f, 1 s)$ & $(1 f, 1 f)$ \\
5 & 200 & $(2 f, 2 s)$ & $(2 f, 3 f)$ \\
\hline
\end{tabular}

$(m, n)$ indicates the modal number for simply-supported or free boundary conditions [16]. $m$ represents the $x$-direction and $n$ represents the $y$-direction. The suffixes $s$ and $f$ are added to indicate the mode shape that corresponds to the simply-supported and free boundary conditions respectively. The asterisk * indicates that the mode does not show a clear boundary condition but was instead assigned the most probable boundary condition.

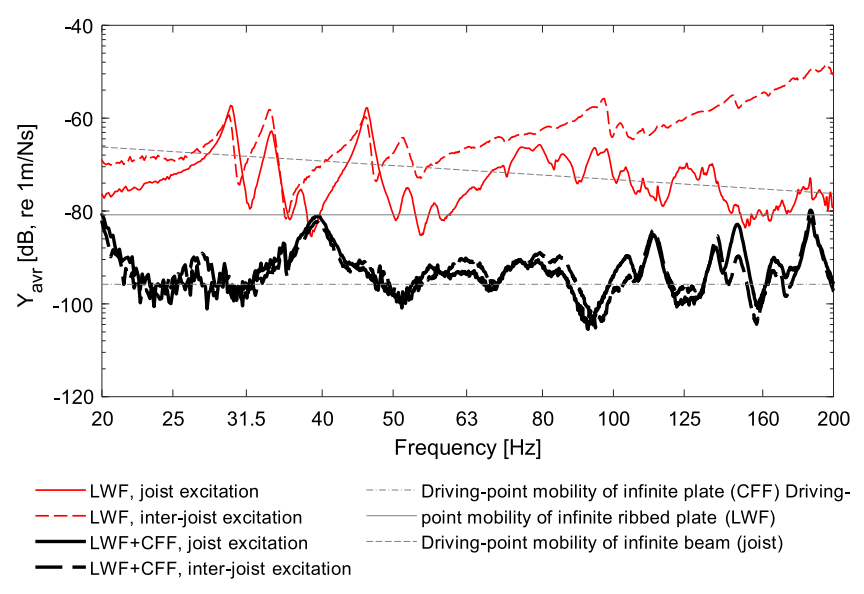

(a)

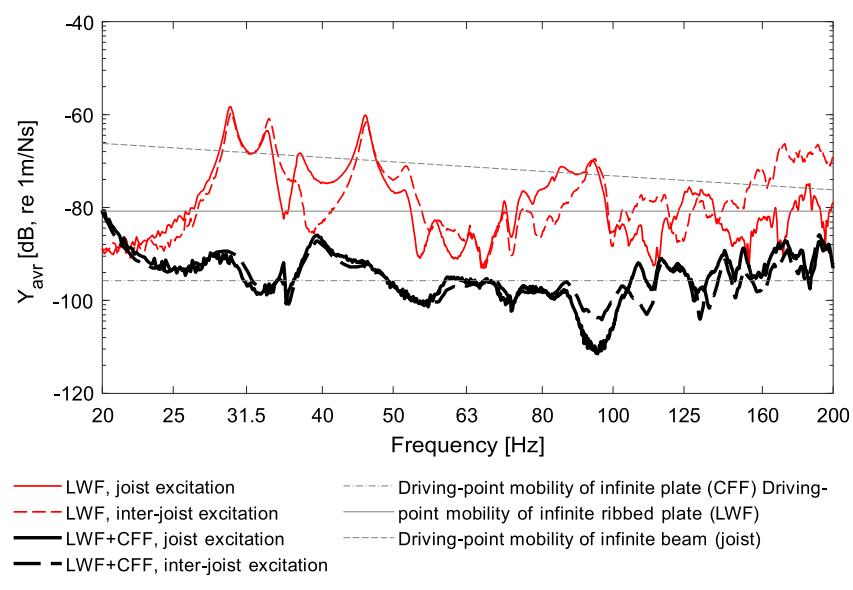

(b)

Figure 10. Magnitude of floor mobilities for joist and inter-joist excitation. The driving point mobility of infinite ribbed plate is calculated based on the method proposed in Section $3(g)$, Chapter IV of [19]. (a) Magnitude of driving-point mobility. (b) Magnitude of transfer mobility. The impact and receiver positions are shown in Figure 3.

affected. As for the LWF + CFF, due to resonance between the floor and room mode the impact sound level is high around $26 \mathrm{~Hz}$. Otherwise, the presence of the body load does not cause any changes on the coupled floor.

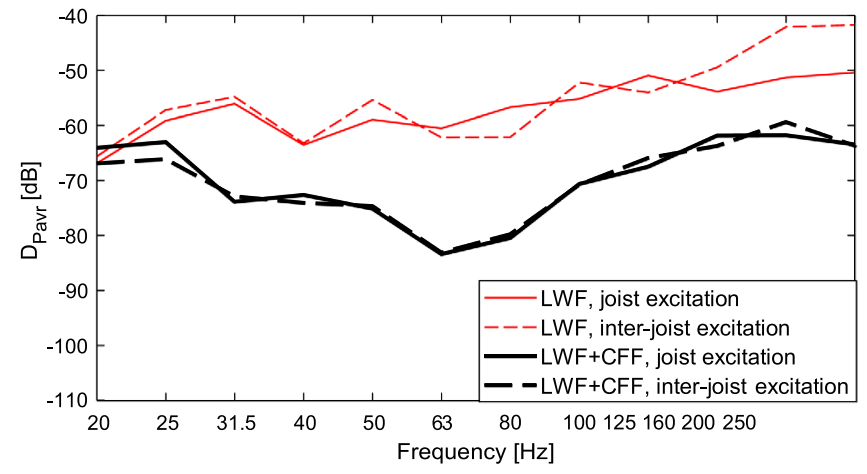

Figure 11. Sound pressure level normalized by impact force for joist and inter-joist excitation.

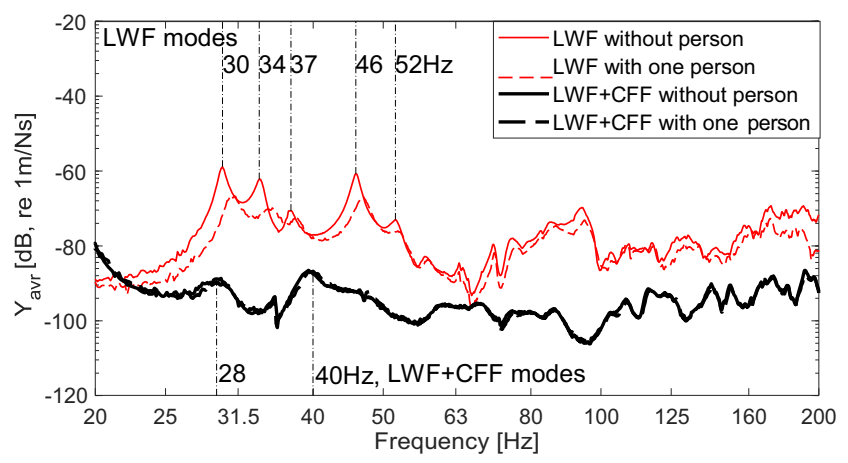

Figure 12. Magnitude of transfer mobility of both LWF and $\mathrm{LWF}+\mathrm{CFF}$ with and without human body load. The impact and receiver positions are shown in Figure 3.

\subsection{Heel-drop excitation}

The frequency spectra of the heel drop on both floors, as measured by the IFP, are shown in Figure 14 together with the spectra from the impact hammer. Differences are observed for both excitation types on both types of floorings, due to the different mobility ratios between the source and the structure. More importantly, while the hammer expectedly yields a rather flat response in the considered frequency range, the heel drop displays a sloped response, with low frequencies reaching up to a $40 \mathrm{~dB}$ higher force compared to the hammer excitation. 


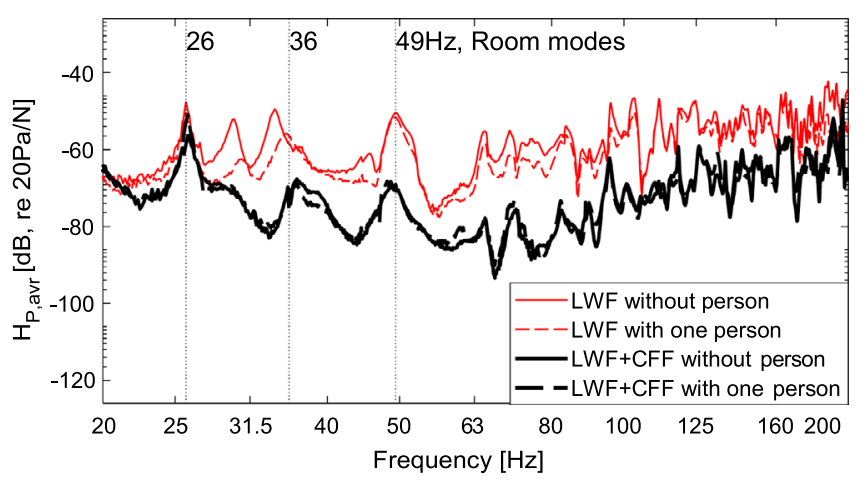

Figure 13. Magnitude of impact sound transfer function of both LWF and LWF + CFF with and without human body load. The impact and receiver positions are shown in Figure 3.

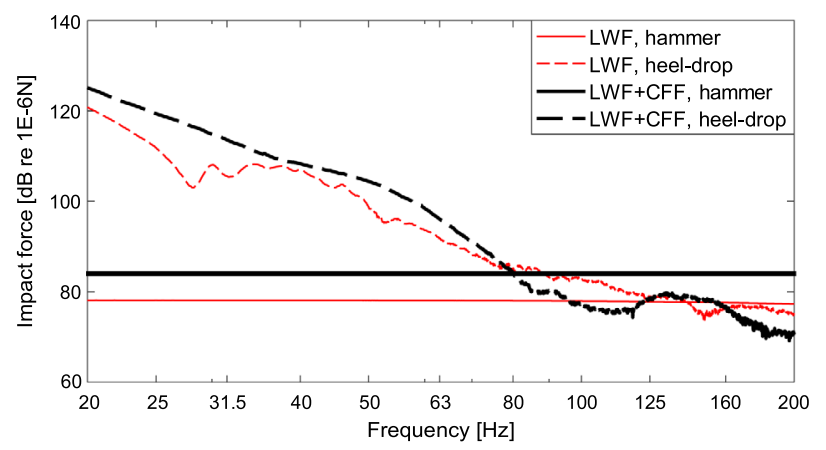

Figure 14. Impact force given by impact hammer and heel drop on both LWF and LWF + CFF.

Figure 15 shows the transfer mobility responses of both types of floors as excited by both impact hammer and heel drop. Comparing the two methods, the excitation via heel drop results in an up to $10 \mathrm{~dB}$ attenuation in the whole inspected frequency range and a decrease in the natural frequencies for both types of floors. For instance, for LWF, the modes at 30, 34 and $37 \mathrm{~Hz}$ have been shifted to 28, 32 and $36 \mathrm{~Hz}$ respectively. The same effect is also observed for the modes of LWF + CFF, for example, at $28 \mathrm{~Hz}$ and $40 \mathrm{~Hz}$.

Figure 16 shows the transmitted impact sound normalized by the respective input forces of both excitations and floor types. While the transfer mobility of the floor demonstrates attenuation when comparing the heel drop to the impact hammer excitation, the same attenuation is not observed for the sound transmitted to the room below the floor. The eigenfrequencies of the floors are still shifted but not for the room modes at 26, 36 and $49 \mathrm{~Hz}$. These room modes were identified via COMSOL simulations. The impact sound transfer function is also compared in terms of one-third-octave bands. The two excitation methods exhibit similar patterns within the frequency range of interest. Nonetheless, a difference of up to $6 \mathrm{~dB}$ can be found in some bands due to the attenuation of vibrations and the shifts of the natural frequencies, e.g., for the $\mathrm{LWF}+\mathrm{CFF}$ below $50 \mathrm{~Hz}$.

Interestingly, the results for the heel-drop test contrast with the results obtained with a standing human (see

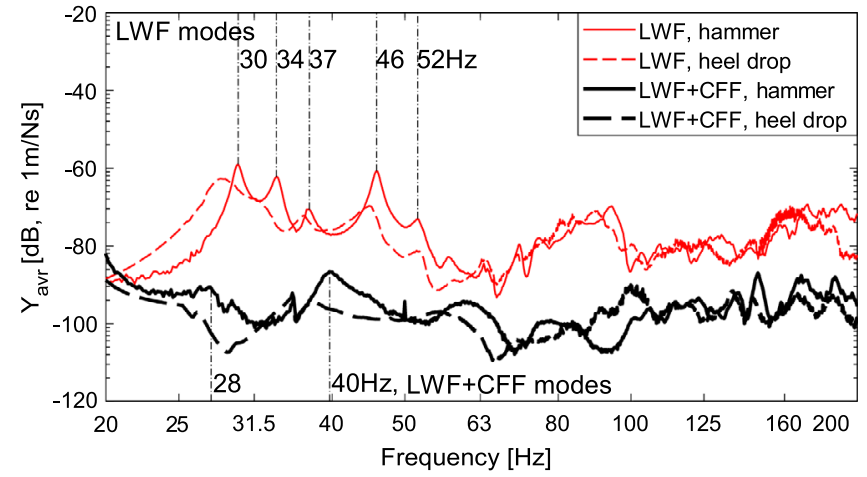

Figure 15. Magnitude of transfer mobilities of both LWF and LWF + CFF when excited by impact hammer and heel drop. The impact and receiver positions are shown in Figure 3.

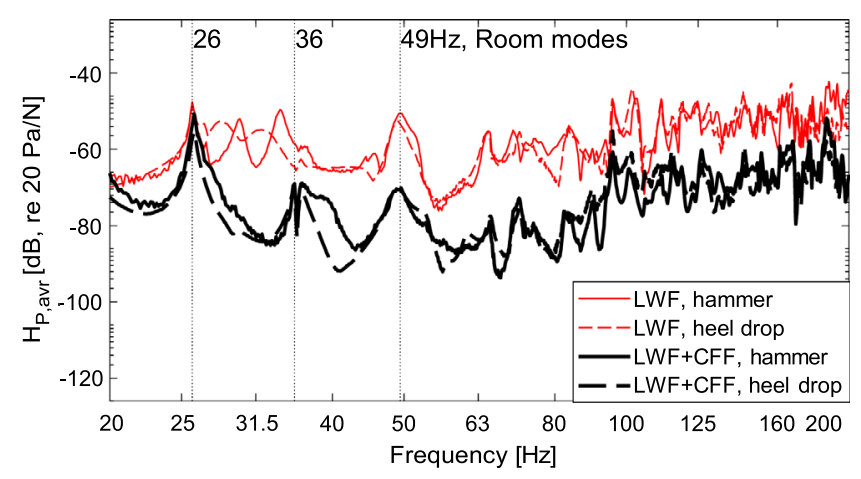

Figure 16. Magnitude of impact sound transfer function of both LWF and LWF + CFF when excited by impact hammer and heel drop. The impact and receiver positions are shown in Figure 3.

Figs. 12 and 15). This difference might be attributed to the dynamic characteristics of the human body which vary depending on the type of motion [13]. Nevertheless, both types of body movements, standing and providing intensive heel drops, reduce the floor vibration and shift the natural modes but do not change the acoustic response of the room modes.

\section{Discussion}

The suitability of the heel-drop excitation as a source for impact sound measurements can be discussed based on the transmitted sound to the room below the floor systems. As shown in Figures 16 and 17, the response of heel drop is very similar to the impact hammer except for the floor mode shift due to human presence.

To further demonstrate the capability of heel-drop excitation, the coherence and signal-to-noise ratio (SNR) are shown in Figures 18a and 18b respectively. Compared to impact hammer excitation, heel drop appears to be disadvantageous only from $80 \mathrm{~Hz}$ onwards in terms of coherence (coherence values lower than 0.75 are commonly considered poor for floor response tests [5]), and $125 \mathrm{~Hz}$ onwards in 


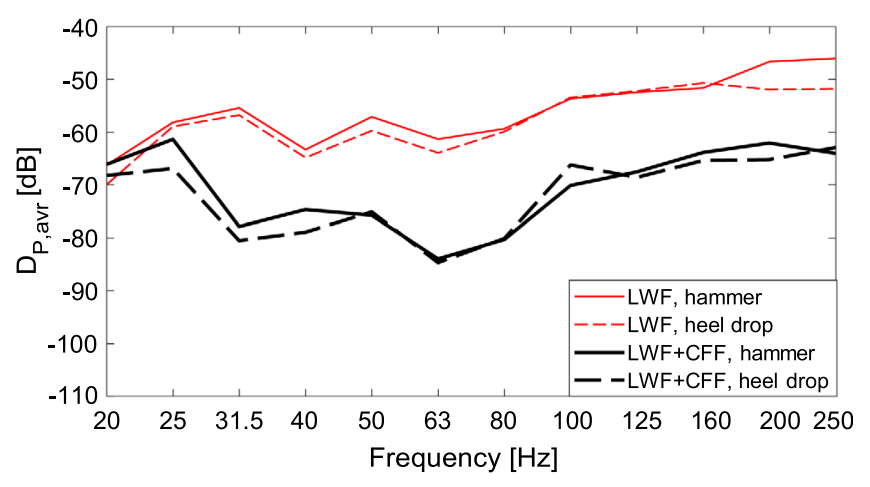

Figure 17. One-third-octave-band level of the impact sound normalized by force. The impact and receiver positions are shown in Figure 3.

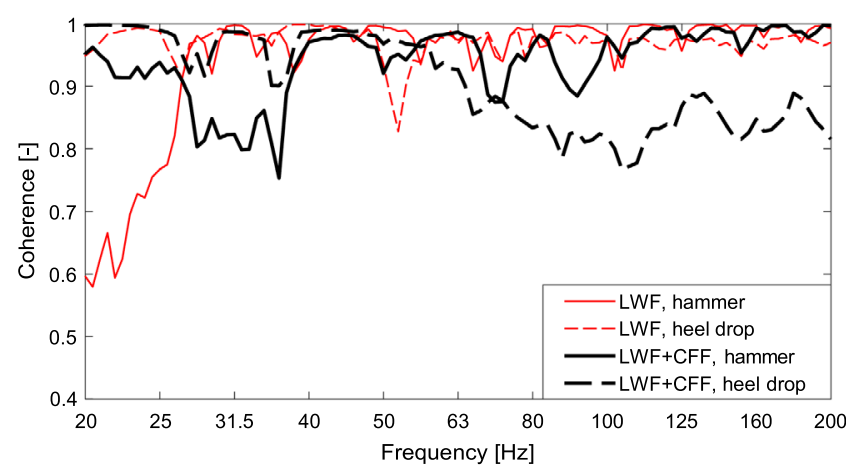

(a)

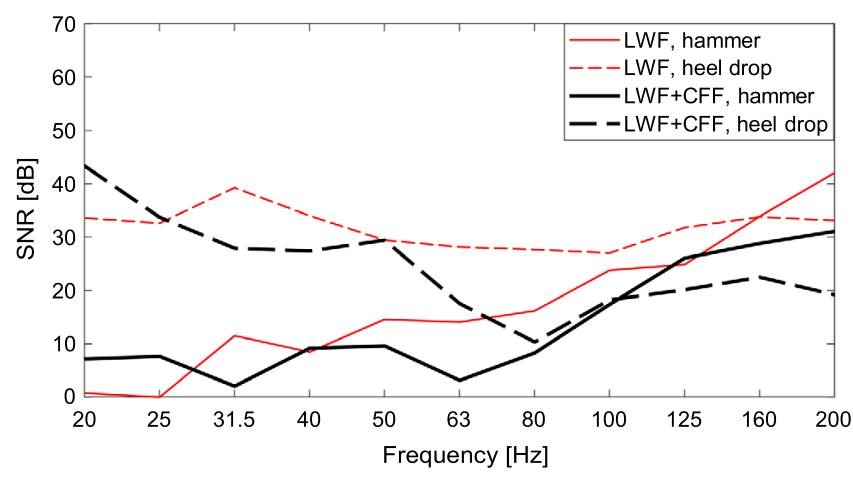

(b)

Figure 18. Coherence and signal-to-noise ratio of the measurements. (a) Coherence of the floor mobility measurements. (b) Signal-to-noise ratio of the impact sound measurements in the receiving room.

terms of SNR for the LWF + CFF. However, it demonstrates superior performance in the low frequency range (below $40 \mathrm{~Hz}$ ). Taking both its advantages and disadvantages into account, the gain for the low frequencies is worth the trade-off considering the slightly worse but still acceptable performance for the higher frequencies frequency. This suggests that heel-drop excitation can be an adequate tool for the frequency range of below $40 \mathrm{~Hz}$ and is worth consideration for measurements that require high coherence and SNR in this frequency range. However, it also needs to be pointed out that only one type of modal hammer was used in the current experiments. Since a larger input force level will lead to a difference in the coherence and SNR results [5], a future study comparing the heel-drop excitation to other heavier and larger modal hammers will be valuable.

On a side note, the use of the transfer function in the impact sound analysis (as shown in Fig. 16) also offers a view on how the LWF experiencing the reduction of the mobility due to the floating layer. The floating concrete panel shifts the LWF natural frequencies and reduces the vibration down to $20 \mathrm{~dB}$ with the only exception at $26 \mathrm{~Hz}$. At $26 \mathrm{~Hz}$, a strong resonance between the floor mode and the room mode happens, remaining the same level of transferred impact sound for the LWF + CFF as the LWF. This indicates avoiding the resonance between the floor and the room is crucial when applying a floating system on a lightweight floor to improve the low-frequency impact sound for a specific room.

\section{Conclusion}

The vibrational behavior of LWF and $\mathrm{LWF}+\mathrm{CFF}$ floors are measured by means of transfer functions. The low-frequency vibration of the LWF is dominated by the natural mode due to simply-supported boundary conditions. When the CFF layer is added on top of the LWF, both layers exhibit a mixture of simply-supported and free conditions.

An impact force plate (IFP) was constructed to measure the input forces using a heel-drop excitation. The device allows the use of the transfer function technique to characterize the floors and demonstrates linearity in the frequency range of interest $(<200 \mathrm{~Hz})$. Comparing the impact hammer and heel-drop excitation, the former has a flat frequency response while the latter has higher energy in the low frequencies, which gives a better SNR below $63 \mathrm{~Hz}$. This suggests that the use of heel-drop excitation is comparable to using an impact hammer in identifying the impact sound and thus evaluating the impact sound performance of the floor via the transfer function.

When the LWF is under a load of a standing human, the vibration of the floor is reduced and the natural frequencies increase. If a human performs a heel-drop excitation, the LWF behaves differently such that the damping increases, and the natural frequencies decrease. The two scenarios show different floor responses due to the different interactions between the human and the floor. However, in any case, the natural frequencies of the room modes and the amplitude of impact-sound response function at these room modes do not show obvious changes regardless of the floorhuman interactions. Comparing to the impact-hammer test, the heel-drop test influences the spectra of the resulting impact-sound transfer function but does not obviously affect the overall transmitted sound pressure level in the room. 


\section{Acknowledgments}

Vloerenbedrijf Van Rijbroek is gratefully acknowledged for supplying the floors used in the present study, and for their support and their time in general. We also thank our colleague Arnold Koopman from Level Acoustics \& Vibration, who provided insight and expertise that greatly assisted the research.

\section{Conflict of interest}

Authors declared no conflict of interests.

\section{References}

1. C. Johansson: Low-frequency impact sound insulation of a light weight wooden joist floor. Applied Acoustics 44, 2 (1995) 133-147.

2. P. Sipari: Sound insulation of multi-storey houses - a summary of Finnish impact sound insulation results. Building Acoustics 7, 1 (2000) 15-30.

3. W.E. Blazier, R.B. DuPree: Investigation of low-frequency footfall noise in wood-frame, multifamily building construction. The Journal of the Acoustical Society of America 96, 3 (1994) 1521-1532.

4. N. Amiryarahmadi, W. Kropp, K. Larsson: Identification of low-frequency forces induced by footsteps on lightweight floors. Acta Acustica United With Acustica 102, 1 (2016) 4557.

5. E. Gerretsen, D. Bard, B. Zhang, B. Ingelaere: Net-acoustics for timber based lightweight buildings and elements, in COST Action FP0702. 2012.

6. C. Hopkins: Sound Insulation. Routledge, 2012.

7. H. Chung, G. Dodd, G. Emms, K. McGunnigle, G. Schmid: Maximising impact sound resistance of timber framed floor/ceiling systems, in Final Report-FWPRDC Project PN04. 2005.

8. N. Labonnote, A. Rønnquist, K.A. Malo: Prediction of material damping in timber floors, and subsequent evaluation of structural damping. Materials and Structures 48, 6 (2015) 1965-1975.

9. A.N. e Sousa, B. Gibbs: Low frequency impact sound transmission in dwellings through homogeneous concrete floors and floating floors. Applied Acoustics 72, 4 (2011) 177-189.

10. B.J. Schwarz, M.H. Richardson: Experimental modal analysis. CSI Reliability Week 35, 1 (1999) 1-12.

11. F. Schöpfer, C. Hopkins, A.R. Mayr, U. Schanda: Measurement of transmission functions in lightweight buildings for the prediction of structure-borne sound transmission from machinery. Acta Acustica United With Acustica 103, 3 (2017) 451-464.

12. P. Reynolds, A. Pavic: Impulse hammer versus shaker excitation for the modal testing of building floors. Experimental Techniques 24, 3 (2000) 39-44.

13. B. Folz, R.O. Foschi: Coupled vibrational response of floor systems with occupants. Journal of Engineering Mechanics 117, 4 (1991) 872-892.

14. U. Schanda, H.-M. Tröbs, R. Völtl, P. Becker: Semiempirical model of the impact force of a walking person in the time domain and generated impact sound spectra, in Internoise 2013. 2018.

15. Iso 10140-5Acoustics - laboratory measurements of sound insulation of building elements - part 7: Requirements for test facilities and equipment. International Organization for Standardization. 2010.

16. P. Gardonio, S. Elliott: Driving point and transfer mobility matrices for thin plates excited in flexure. ISVR Technical Report No 277, 1998.

17. P. Avitabile: Modal testing: A practitioner's guide. John Wiley \& Sons, 2017.

18. A.R. Mayr, B. Gibbs: Point and transfer mobility of pointconnected ribbed plates. Journal of Sound and Vibration 330, 20 (2011) 4798-4812.

19. L. Cremer, M. Heckl, E. Ungar: Structure-borne sound: Structural vibrations and sound radiation at audio frequencies. Springer-Verlag, 2005.

20. S. Zhang, L. Xu, J. Qin: Vibration of lightweight steel floor systems with occupants: modelling, formulation and dynamic properties. Engineering Structures 147 (2017) 652-665.

21. B. Ellis, T. Ji, BRE: Human-structure interaction in vertical vibrations. Proceedings of the Institution of Civil EngineersStructures and Buildings 122, 1 (1997) 1-9.

Cite this article as: Qin Y, Tan JJ \& Hornikx M. 2020. Experimental investigation of the low-frequency impact sound transfer function of lightweight wooden floors via heel drops. Acta Acustica, 4, 23. 